\title{
Effects of earthworm extract on the lipid profile and fatty liver induced by a high-fat diet in guinea pigs
}

\author{
Zhenhan Deng ${ }^{1,2,3 \#}$, Shanshan Gao ${ }^{4 *}$, Yunfei An ${ }^{5}$, Yong Huang ${ }^{1}$, Haifeng Liu ${ }^{1}$, Weimin Zhu ${ }^{1}$, Wei Lu ${ }^{1}$, \\ Miao $\mathrm{He}^{2}$, Wenqing $\mathrm{Xie}^{2}$, Dengjie $\mathrm{Yu}^{2}$, Yusheng $\mathrm{Li}^{2}$ \\ ${ }^{1}$ Department of Sports Medicine, Key Laboratory of Tissue Engineering of Shenzhen, Shenzhen Second People's Hospital/the First Affiliated \\ Hospital of Shenzhen University Health Science Center, Shenzhen, China; ${ }^{2}$ Department of Orthopaedics, Xiangya Hospital, Central South \\ University, Changsha, China; ${ }^{3}$ School of Medicine, Shenzhen University, Shenzhen, China; ${ }^{4}$ Division of Cardiology, University of Colorado Anschutz \\ Medical Campus, Aurora, CO, USA; ${ }^{5}$ Department of Animal Science, College of Animal Science and Technology, Hunan Agricultural University, \\ Changsha, China \\ Contributions: (I) Conception and design: Z Deng, Y Li; (II) Administrative support: Z Deng, S Gao, Y An, Y Li; (III) Provision of study materials \\ or patients: Z Deng, S Gao, Y An, Y Huang, H Liu, W Zhu, W Lu, Y Li; (IV) Collection and assembly of data: Z Deng, S Gao, Y An, Y Huang, H \\ Liu, M He, W Xie, D Yu, Y Li; (V) Data analysis and interpretation: Z Deng, S Gao; (VI) Manuscript writing: All authors; (VII) Final approval of \\ manuscript: All authors. \\ \#These authors contributed equally to this work. \\ Correspondence to: Dr. Yusheng Li. Department of Orthopaedics, Xiangya Hospital, Central South University, Changsha 410008, China. \\ Email: liyusheng@csu.edu.cn.
}

Background: Non-alcoholic fatty liver disease (NAFLD), characterized by the accumulation of excess fat in the liver in people who consume little or no alcohol, is becoming increasingly common around the world, especially in developed countries. Extracts from earthworms have been used as alternative therapies for a variety of diseases but not in NAFLD. Therefore, the aim of this study was to investigate the effect of earthworm extract (EE) on diet-induced fatty liver disease in guinea pigs.

Methods: EE was extracted, and the effect of EE on the lipid levels and liver damage in guinea pigs fed a high-fat diet (HFD) was assessed. Thirty male guinea pigs at 3 weeks of age were allocated equally to five groups, namely, chow diet, HFD, and HFD with different dosages $(0.3,1.4$ and $6.8 \mu \mathrm{g}$ per $\mathrm{kg}$ bodyweight per day) of EE for 4 weeks, and their body weight was monitored throughout the experiment. Liver tissues were examined for gross morphology and histology. Serum levels of total cholesterol (TC), triglycerides (TG), low-density lipoprotein cholesterol (LDL-C), alanine transaminase (ALT) and aspartate aminotransferase (AST) were determined using an autoanalyser.

Results: HFD induced NAFLD in guinea pigs. HFD-fed guinea pigs that received EE treatment showed milder increases in the serum levels of TC, TG and LDL-C, as well as in the body weight growth rate, compared to the HFD group without EE supplementation. EE intervention reduced the number of lipidcontaining hepatocytes, hepatocellular ballooning and sinusoidal distortion in the liver in HFD-fed animals. ALT in serum was significantly elevated by HFD. No statistically significant difference in ALT levels was found between the chow diet group and the HFD group with EE treatment.

Conclusions: This study demonstrates that the administration of EE suppressed the induction of serum TC, TG and LDL-C in response to HFD. EE also reduced liver damage in HFD-fed guinea pigs. These findings suggest that EE has alleviating effects on dyslipidaemia and liver damage associated with NAFLD.

Keywords: Hyperlipidaemia; earthworm extract (EE); lipid metabolism; fatty liver disease

Submitted Jul 16, 2020. Accepted for publication Nov 27, 2020.

doi: $10.21037 /$ atm-20-5362

View this article at: http://dx.doi.org/10.21037/atm-20-5362 


\section{Introduction}

Non-alcoholic fatty liver disease (NAFLD) is a major public health concern due to its worldwide prevalence (1). To date, there are no effective therapeutic treatments for NAFLD except weight loss (2). Consumption of a highfat diet (HFD) increases the chance of NAFLD (3), and patients with NAFLD often have clinical features such as dyslipidaemia (4), obesity (5), and hypertension (6). Previous studies have shown that guinea pigs fed a HFD for 4 weeks exhibit obesity, dyslipidaemia and liver damage $(7,8)$. Due to the resemblance of symptoms achieved by HFD in guinea pigs to those in humans, HFD-fed guinea pigs have been considered a suitable animal model to study the mechanisms underlying the development of NAFLD $(8,9)$.

Alanine aminotransferase (ALT) and aspartate aminotransferase (AST) are two enzymes mostly found in the liver (10). When liver damage occurs, ALT and AST are released into the bloodstream (10), so elevated levels of ALT and AST may indicate liver damage and, as a result, have been used as standard tests to monitor liver diseases, including fatty liver disease (11).

Dyslipidaemia refers to blood lipid levels that are higher or lower than the normal range (12). The dyslipidaemia present in NAFLD is usually hyperlipidaemia, characterized by elevated serum triglyceride (TG) and low-density lipoprotein cholesterol (LDL-C) levels and decreased level of high-density lipoprotein cholesterol (HDL-C) (4). Synthesized by the liver, TG is the major form of fatty acid storage and transport in our body, and the hepatic accumulation of triglycerides represents a hallmark of NAFLD (13). LDL carries cholesterol to cells under physiological conditions. However, high LDL-C levels contribute to lipid build-up in blood vessels, which triggers the inflammatory response and accelerates the lipid accumulation process (14). Hence, LDL-C has been regarded as a "bad" cholesterol in general. High-density lipoprotein cholesterol (HDL), on the other hand, not only plays a critical role in lipid transport from peripheral tissues to the liver for lipid metabolism but also possesses anti-inflammatory properties (15). Therefore, HDL-C is considered a cholesterol that is beneficial to health.

Several medicines, such as statins, ezetimibe and PCSK9 inhibitors, have been developed to lower the levels of TG and/or LDL-C and induce the elevation of HDL-C (16). However, side effects including muscle aches, drowsiness, and high blood sugar with these medications have been described (17-19). Adverse hepatic effects are among the most frequently observed side effects with statin utilization (20). Similarly, cases of patients who developed severe hepatic side effects following ezetimibe treatment have been reported $(21,22)$.

The use of earthworms (Lumbricina) as a medicine dates back hundreds of years. Several studies have evaluated the influence of earthworm-derived components on haematological and blood chemistry parameters in animals and healthy human subjects, finding no significant adverse effects $(23,24)$. In terms of the association between earthworm-derived components and lipid metabolism, Kawakami et al. found that Zucker diabetic fatty (ZDF) rats fed composite earthworm powder display attenuated hepatic lipid accumulation (25). Balamurugan et al. demonstrated that oral administration of EE ameliorated hepatic injury induced by paracetamol in rats. In their study, EE treatment increased the levels of hepatic antioxidant activities and decreased the levels of AST and ALT, indicating that components from EE may have a hepatoprotective effect, possibly by preventing oxidative group formation (26). Additionally, another study showed that rats fed dried earthworm powder exhibited a milder increase in the lipidperoxidative marker thiobarbituric acid reactive substance (TBARS) and a lower reduction in antioxidant enzyme activities in the presence of alcohol-induced hepatic damage, indicating a potential impact of earthworm-derived components on antioxidant enzyme synthesis (27). These findings suggest that EE may improve the lipid profile and liver function in HFD-induced NAFLD guinea pigs.

Parwanto et al. performed fractionation and characterization of proteins based on molecular weight and showed that the majority of proteins isolated from the earthworm Lumbricus rubellus have molecular weights ranging from 5 to $100 \mathrm{kDa}$ (28). Therefore, we hypothesize that the $3-100 \mathrm{kDa}$ fraction of EE will improve the lipid profile and liver function in HFD-induced NAFLD guinea pigs. We expect to see decreased serum levels of TC, TG and LDL-C; increased levels of HDL-C; and reduced HFD-induced liver injury with EE supplementation in a dose-dependent manner. We present the following article in accordance with the ARRIVE reporting checklist (available at http://dx.doi.org/10.21037/atm-20-5362).

\section{Methods}

The study was approved by the Ethics Committee at the First Hospital Affiliated to Shenzhen University (SZU), Shenzhen Second People's Hospital (No. 20170135), 
Table 1 Components of diets used in the experiments

\begin{tabular}{lcc}
\hline Components & Chow diet $(\mathrm{g} / 100 \mathrm{~g})$ & High-fat diet $(\mathrm{g} / 100 \mathrm{~g})$ \\
\hline Protein & 19.3 & 19.3 \\
Fat mix & 3.2 & 3.2 \\
Mineral mix & 8.2 & 8.2 \\
Vitamin mix & 1.1 & 1.1 \\
Fiber & 11.8 & 11.8 \\
Cholesterol & 0.0 & 0.1 \\
Lard & 0.0 & 10.0 \\
\hline
\end{tabular}

granted by the institutional/regional/national ethics/ committee/ethics board of the Institutional Animal Care and Use Committee of SZU, in compliance with national/ institutional guidelines for the care and use of animals.

\section{Isolation of EE}

Live Indian blue earthworms (Perionyx excavates) raised in the laboratory were rinsed with tap water and kept in water overnight to purge the sand from the earthworms. Subsequently, the earthworms were sonicated briefly to remove slime, followed by tissue homogenization. The homogenized solution was sonicated again and centrifuged at $8,000 \mathrm{rpm}$ at $4{ }^{\circ} \mathrm{C}$ to collect the supernatant. Protein was purified by ammonium sulfate precipitation (saltingout), dissolved in phosphate buffered saline (PBS), and then loaded onto centrifugal units with a $100-\mathrm{kDa}$ nominal molecular weight cut-off (NMWCO). After the first ultracentrifugation, the fraction that passed through the filter was collected. The second ultracentrifugation was performed using centrifugal units with $3-\mathrm{kDa} \mathrm{NMWCO}$, and the fluid retained by the filter was collected and ready to be used in the experiments.

\section{Animals}

Thirty 3-week-old male Hartley guinea pigs (Topgene Biotechnology, Wuhan, China) were housed in a temperature-controlled room $\left(20-24{ }^{\circ} \mathrm{C}\right)$ with a 12 -h light/ dark cycle. Food and water were provided ad libitum. The animals were randomized into five groups $(\mathrm{n}=6)$ and fed a normal chow diet, a HFD containing $0.1 \%$ cholesterol and $10 \%$ lard, and a HFD with low $(0.3 \mu \mathrm{g} / \mathrm{kg} / \mathrm{d})$, medium $(1.4 \mu \mathrm{g} / \mathrm{kg} / \mathrm{d})$ or high $(6.8 \mu \mathrm{g} / \mathrm{kg} / \mathrm{d})$ doses of EE for 4 weeks.
The components of the chow diet and the HFD used in this study are listed in Table 1. EE was orally administered to the animals. The body weight of each guinea pig was recorded throughout the experiment. Body weight gain was calculated using the following formula: weight gain $(\%)=$ 100× (measured weight - initial weight)/initial weight. At the end of the experiment, all the animals were fasted for $12 \mathrm{~h}$ before being sacrificed.

\section{Histology}

A $1 \mathrm{~cm}^{2}$ central piece from the right lobe of the liver was taken from each sacrificed animal, fixed in $4 \%$ paraformaldehyde (PFA) solution overnight, dehydrated and embedded in paraffin. Tissue blocks were sectioned at $5 \mu \mathrm{m}$ thickness and stained with haematoxylin and eosin (HE) for microscopic observation. Ten sections were randomly picked from each liver sample and viewed by 3 different individuals. For each section, 20 randomly selected fields were examined for histology. NAFLD scores based on the histological presentations were calculated as previously described (29).

\section{Biochemical analysis}

Blood was collected from the hearts of animals under general anaesthesia, after which animals were sacrificed by cervical dislocation. Blood samples were centrifuged at 2,500 rpm for $15 \mathrm{~min}$, and the supernatant serum was collected and stored at $4{ }^{\circ} \mathrm{C}$ in the short term. Serum levels of TG, TC, HDL-C, LDL-C, ALT and AST were analysed with an autoanalyser (Modular E170; Roche, Basel, Switzerland).

\section{Liver index}

After all the animals were sacrificed, the livers were removed and weighed. The liver index was calculated as the ratio of liver to body weight.

\section{Statistical analysis}

All statistical analyses were performed using SPSS 17.0 software (Chicago, IL, USA). Weekly body weight data were analysed by repeated measures ANOVA with diet, time, experiment and their interactions as factors. Analyses of all the other data were performed using one-way ANOVA followed by the Newman-Keuls post hoc test. Differences with a $\mathrm{P}$ value $<0.05$ were considered statistically significant. 

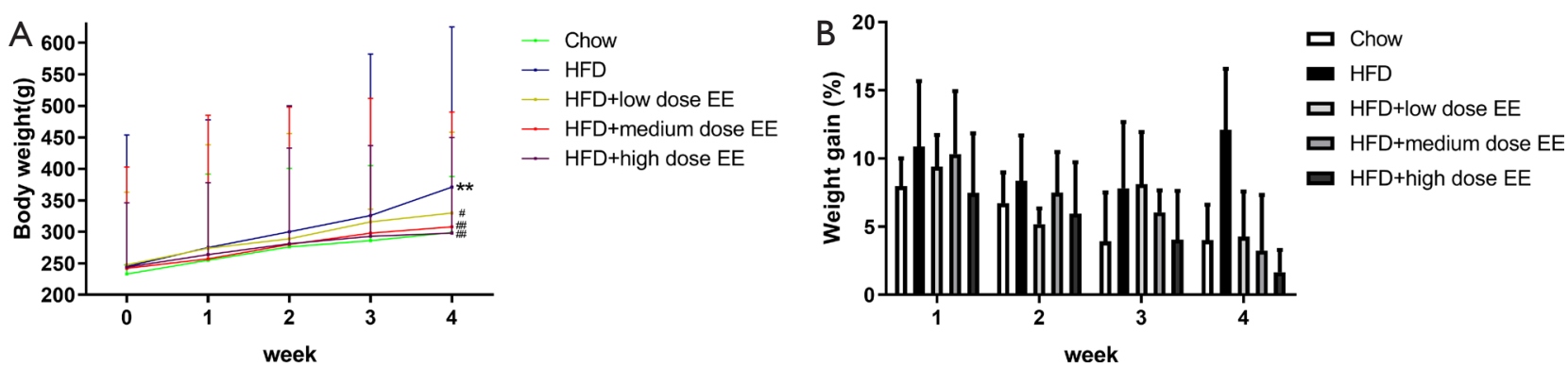

Figure 1 Effect of EE on body weight (A) and body weight gain (B) in guinea pigs fed a chow diet, a HFD, or a HFD with EE supplementation for 4 weeks. Data shown are the mean $\pm \mathrm{SD}$ ( $\mathrm{n}=6$ per group). **, $\mathrm{P}<0.01$ vs. chow group; ${ }^{\#}, \mathrm{P}<0.05 ;{ }^{\#}, \mathrm{P}<0.01$ vs. $\mathrm{HFD}$ model group.

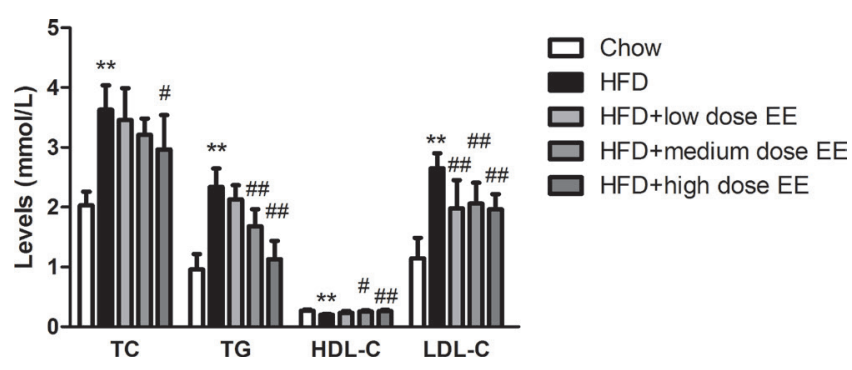

Figure 2 Effect of EE on the lipid profile in guinea pigs. Serum levels of TC, TG, HDL-C and LDL-C were measured in animals fed a chow diet, a HFD or a HFD with EE supplementation for 4 weeks. Data shown are the mean $\pm \mathrm{SD}$ ( $\mathrm{n}=6$ per group). **, $\mathrm{P}<0.01$ vs. chow group; ${ }^{*}, \mathrm{P}<0.05,{ }^{\#}, \mathrm{P}<0.01$ vs. HFD model group.

\section{Results}

\section{Effect of EE on body weight in HFD-fed guinea pigs}

No significant differences were observed in body weights between all the groups of animals at weeks 1,2 or $3(\mathrm{P}>0.05)$. At week 4, the HFD group showed a higher body weight than the control group $(\mathrm{P}<0.01)$, while all the animals with $\mathrm{EE}$ treatment displayed body weights lower than the HFD model group but not the control animals $(\mathrm{P}<0.05$, Figure $1 A$ ). Weekly body weight gain per guinea pig did not differ among all the groups throughout the experimental period $(\mathrm{P}>0.05)$; however, the HFD group constantly showed slightly higher weight gain than the other groups from week 1 to 4 , and mild dose-dependent reductions in weight gain were observed in the EE-treated animals compared to the body weight gain of the HFD group at weeks 3 and 4 (Figure 1B). These results suggest that oral administration of $\mathrm{EE}$ can reduce the body weight gain caused by HFD in guinea pigs in a dose-dependent manner.

\section{Effect of EE on the serum lipid profile}

The serum lipid profile obtained from blood samples at the end of the experiment showed a prominent increase in TC, TG and LDL-C in guinea pigs fed a HFD without EE treatment compared to the control group, while the level of HDL-C dropped significantly in the HFD group without $\mathrm{EE}$ treatment compared to animals on a chow diet. A suppressed increase in TC levels was found in the HFD group with medium- and high-dose EE treatment but not in the low-dose EE-treated animals. Similarly, a decrease in TG was observed in guinea pigs orally administered medium and high doses of EE. There was less decrease in HDL-C caused by HFD in medium- and high-dose EE-treated animals. Additionally, all the animals fed with EE showed lower levels of LDL-C than the HFD group $(\mathrm{P}<0.01)$, although no dose-dependent effect was observed (Figure 2). These results indicate that $\mathrm{EE}$ can negatively regulate the induction of TG and LDL-C and abolish the HDL-C-lowering effect in HFD-fed guinea pigs.

\section{Effect of EE on the liver index}

At the end of the experiment, the HFD group had the highest liver index among all the groups. All EE-treated animals displayed lower liver indexes than the HFD group (Figure 3).

\section{EE supplementation ameliorated HFD-induced morphological changes in livers}

Morphological examination of livers revealed that HFD feeding for 4 weeks led to several gross morphological alterations. Livers from the control group were reddish- 


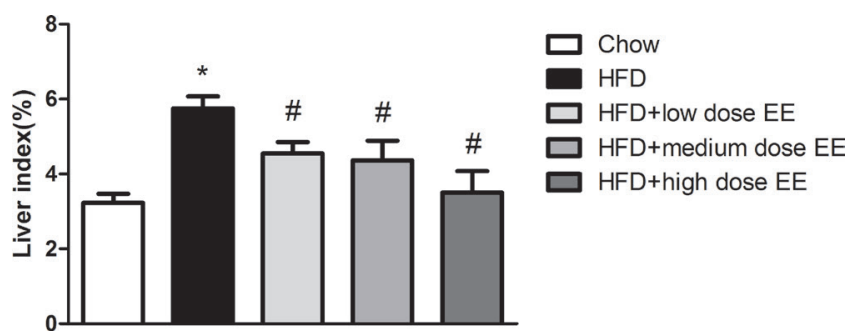

Figure 3 Effect of EE on the liver index in guinea pigs fed a chow diet, a HFD, or a HFD with EE supplementation for 4 weeks. Data shown are the mean $\pm \mathrm{SD}\left(\mathrm{n}=6\right.$ per group). ${ }^{*}, \mathrm{P}<0.05$ vs. chow group; " $\mathrm{P}<0.05$ vs. HFD model group. brown in colour, had smooth surfaces and sharply delineated inferior margins. In contrast, livers from guinea pigs fed a HFD showed increased size, lightening in colour, rounded inferior margins and surface nodularity. Nevertheless, animals treated with EE showed ameliorated hepatic injury (Figure $4 A$ ), suggesting that EE can partially reverse the fatty liver symptoms caused by HFD. Histological analysis (Figure $4 B$ ) showed that guinea pigs in the control group had clearly demarcated and uniform hepatic sinusoids; no lipid vacuoles were observed in the hepatocytes surrounding the central vein of hepatic

A
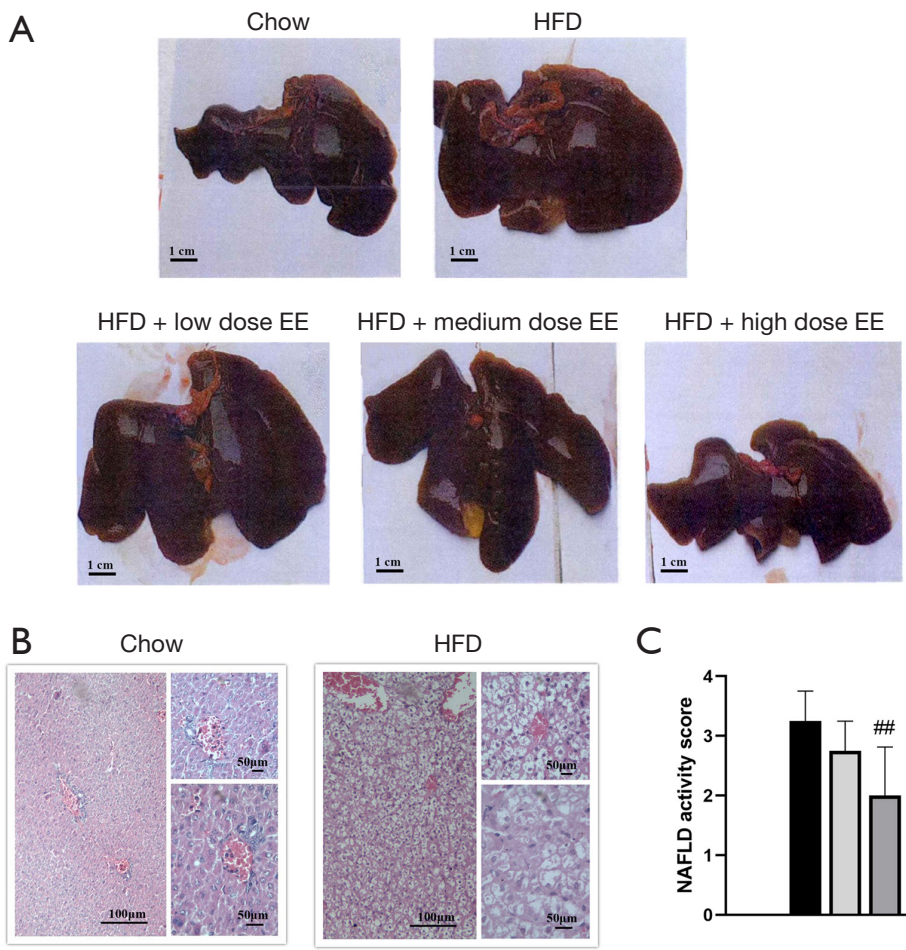

C
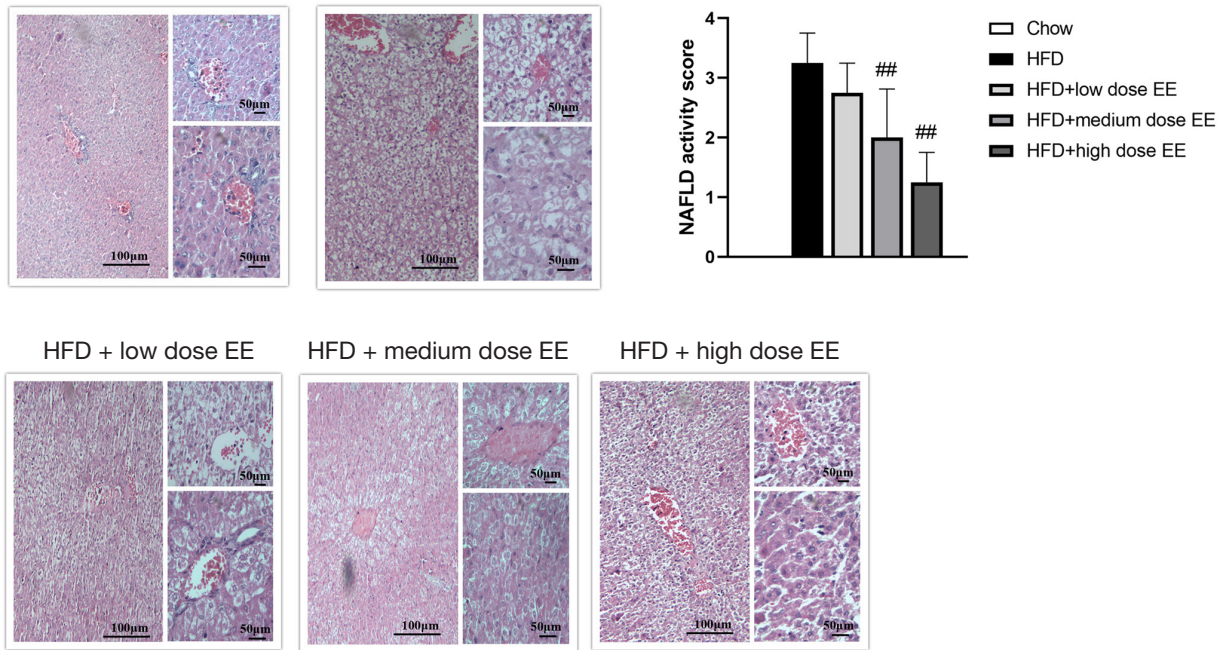

Figure 4 Oral administration of EE attenuates liver injury and hepatic lipid accumulation in response to HFD. (A) Liver gross morphology. (B) Representative pictures of the liver stained with haematoxylin and eosin. (C) Non-alcoholic fatty liver disease (NAFLD) activity scores of the liver based on haematoxylin and eosin staining liver images. ${ }^{\text {\#\# }}, \mathrm{P}<0.01 v s$. HFD model group. 

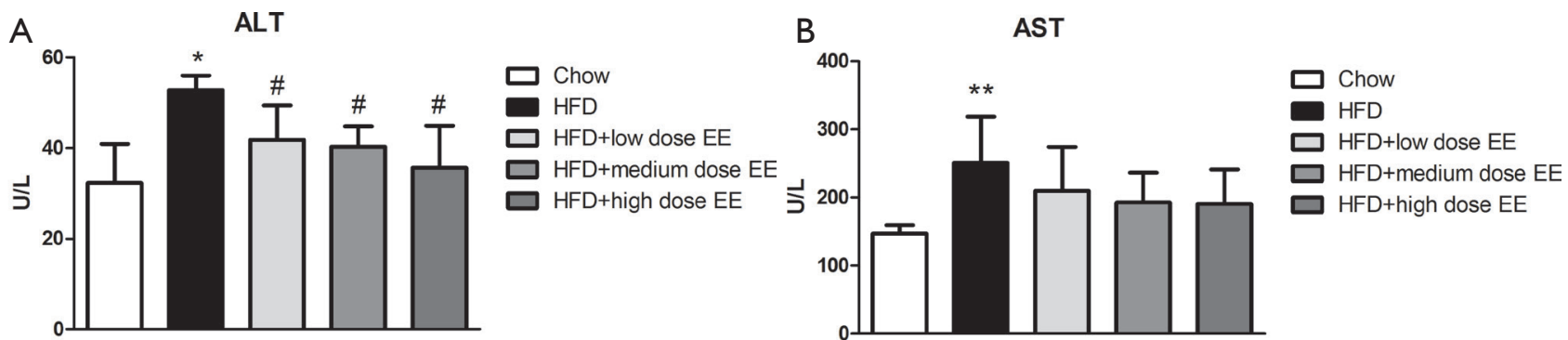

Figure 5 ALT (A) and AST (B) levels of the guinea pigs fed a chow diet, a HFD, or a HFD with EE supplementation for 4 weeks. Data shown are the mean $\pm \mathrm{SD}$ (n=6 per group). *, $\mathrm{P}<0.05$; **, $\mathrm{P}<0.01$ vs. chow group;, $\mathrm{P}<0.05$ vs. HFD model group.

lobules or in the portal canals in control livers. Conversely, animals fed a HFD exhibited sinusoidal distortion, hepatocellular ballooning, and accumulation of lipid droplets in hepatocytes, especially in cells surrounding the central and portal veins. The low-dose EE-treated group showed fewer hepatocytes containing lipid droplets in the portal areas than the HFD group. Notably, mediumdose EE-fed animals showed even fewer lipid dropletfilling hepatocytes around the portal veins than the lowdose group. Guinea pigs fed high-dose EE had uniform sinusoids, morphologically normal hepatocytes in the portal area and much fewer hepatocytes with intracellular fat vacuoles around the central veins. The NAFLD activity score (NAS) was calculated based on the extent of steatosis, lobular inflammation, fibrosis and hepatocellular ballooning for histological evaluation of NAFLD (30). In the HFD model group, the NAS was higher than 3. In HFD animals treated with medium-dose $(1.4 \mu \mathrm{g} / \mathrm{kg} / \mathrm{d})$ and high-dose $(6.8 \mu \mathrm{g} / \mathrm{kg} / \mathrm{d}) \mathrm{EE}$, the NAS were significantly lower than those of the HFD group. These results suggest that oral administration of high-dose $(6.8 \mu \mathrm{g} / \mathrm{kg} / \mathrm{d}) \mathrm{EE}$ can alleviate the morphological alterations in the liver caused by HFD in guinea pigs.

\section{EE supplementation attenuated HFD-induced hepatic damage}

The elevations in ALT and AST serum levels may result from disorders including metabolic disease, cardiovascular disease, and liver disease. Therefore, these tests have been used to assess liver damage and function. Compared to animals fed chow, the HFD group showed elevated serum levels of ALT and AST. Guinea pigs treated with EE exhibited slightly but not significantly reduced AST in comparison with the HFD group $(\mathrm{P}>0.05)$. However, a marked decrease in ALT was observed in the EE-treated group in a dose-dependent manner (Figure 5). These results indicate that HFD-induced increases in ALT and AST can be suppressed by oral administration of EE in guinea pigs.

\section{Discussion}

In the current study, we used guinea pigs fed a HFD as an animal model of NAFLD. We observed that the body weights of guinea pigs fed a HFD, with or without EE treatment, did not show a significant difference compared to the control animals at weeks 1, 2 or 3. Additionally, no difference was found among all the groups of animals in weekly body weight gain during this experiment. In previous studies, scientists found that although HFD does not significantly induce body weight gain in guinea pigs like it does in rats and mice, it often leads to dyslipidaemia and other metabolic symptoms $(31,32)$. In our study, increased serum levels of TC, LDL-C and TG were detected in animals fed a HFD, suggesting that relative hyperlipidaemia was induced. However, because we used 3-week-old guinea pigs at the start of the experiment and the duration of the experiment was 4 weeks, it is difficult to conclude that HFD feeding induced obesity in the animals since the normal body weight ranges of young guinea pigs are not welldefined. Guinea pigs reach sexual maturity between 3 and 4 months, and the body weights of adult guinea pigs are usually between $700 \mathrm{~g}$ and $1,200 \mathrm{~g}$ (33). There is a rapid growth phase in guinea pigs' lives, usually before they reach 2 months old, in which they gain as much as 5 to $7 \mathrm{~g}$ per day when food is provided ad libitum (34). The average daily body weight gain values obtained from all the groups of animals in our study were below $7 \mathrm{~g}$ (data not shown), even in animals fed a HFD. Therefore, even though the guinea pigs fed the HFD had significantly higher body weights than those fed chow at the end of the experiment, we refrained from referring to these animals as overweight/ 
obese. Despite the obesity-resistant phenotype, HFD-fed guinea pigs are a widely used animal model to investigate metabolic syndromes. Yang et al. found that 4weeks of feeding a HFD containing $0.1 \%$ cholesterol and $10 \%$ lard could raise the plasma levels of TC, LDL-C and TG in guinea pigs but not in rats (9). Furthermore, there are many similarities shared by guinea pigs and humans in terms of lipid composition and lipid metabolism. Fernandez et al. found that guinea pigs not only have a lipid profile resembling that of humans but also have LDL as the main carrier of cholesterol in the circulation, similar to humans, which has not been seen in other rodents (7). Additionally, guinea pigs develop NAFLD with key hepatic histological features similar to those observed in humans under the influence of HFD (35), which renders them suitable models to study the pathogenesis of NAFLD.

The reference ranges for plasma lipids in guinea pigs are as follows: TC: $0.00-2.06 \mathrm{mmol} / \mathrm{L}$; TG: $0.74-0.88 \mathrm{mmol} / \mathrm{L}$; LDL-C: $1.45-2.01 \mathrm{mmol} / \mathrm{L}$; and HDL-C: $0.18-0.24 \mathrm{mmol} / \mathrm{L}(36,37)$. In our study, HFD led to physiologically significant elevations of TC $(3.63 \pm 0.41 \mathrm{mmol} / \mathrm{L}), \mathrm{TG}(2.34 \pm 0.31 \mathrm{mmol} / \mathrm{L})$ and LDL-C $(2.65 \pm 0.25 \mathrm{mmol} / \mathrm{L})$ in guinea pigs. In animals treated with high-dose $(6.8 \mu \mathrm{g} / \mathrm{kg} / \mathrm{d}) \mathrm{EE}$, the plasma levels of TC $(2.97 \pm 0.57 \mathrm{mmol} / \mathrm{L})$ and TG $(1.13 \pm 0.31 \mathrm{mmol} / \mathrm{L})$ were significantly lower than those in the HFD-fed group, although they were still considered high levels in guinea pigs. The serum concentration of LDL-C was significantly increased and higher than the reference range in HFD-fed animals, while treatment with all three doses of EE brought the LDL-C level down to normal. HDL-C levels were reduced but remained within the reference range in guinea pigs fed a HFD without EE treatment.

There are two main types of fatty liver disease: alcoholic and non-alcoholic (38). Alcoholic fatty liver disease (AFLD) is commonly found in people who have excessive alcohol intake (39), while the major risk factors for NAFLD include obesity, dyslipidaemia and metabolic syndromes (40). A histological hallmark of NAFLD is the accumulation of lipid vacuoles that have a clear appearance with $\mathrm{H} \& \mathrm{E}$ staining in hepatocytes (41). Hepatocytes take up lipids in the form of free fatty acids (FFAs) from the circulation mainly by facilitated transport, a process mediated by multiple FFA transport proteins (42). The presence of a tiny amount of fat in the liver is considered normal, while a liver with more than $5 \%$ of hepatocytes containing lipids is considered a fatty liver based on the histological criterion for NAFLD diagnosis (43). A HFD has been reported to induce lipid accumulation in hepatocytes surrounding the central veins (44). In addition to the direct effect of a HFD on hepatic lipid accumulation, a HFD leads to insulin resistance, which increases FFA transport to the liver and accelerates the progression of fatty liver disease (45). HFD consumption also promotes oxidative imbalance (46). Yu et al. showed that oxidative stress induced by a HFD impaired the function of hepatocyte nuclear factor $4 \alpha$ $(\mathrm{HNF} 4 \alpha)$, a critical regulator of hepatic lipid homeostasis, thus increasing lipid accumulation in the liver (47). In our study, lipid droplets were mainly observed in hepatocytes around the central veins in guinea pigs fed a HFD, and treatment with EE partially reversed this morphological disruption in the liver.

Indian blue earthworms (Perionyx excavates), commercially available and used in several studies (48-50), serve as a great source of potential therapeutic components. To date, only a few studies have successfully identified lipid-lowering constituents derived from earthworms. Composite powder containing earthworms (CEP) was found to significantly reduce the total lipid content and triglycerides in ZDF rats, but it remains unclear whether components from earthworms are the primary contributors to the lipid-regulating effect observed (25).

Nevertheless, an in vitro assay revealed that CEP possesses plasminogen activator-like activity (25). Plasminogen is the inactive precursor of plasmin, a proteolytic enzyme that breaks down blood clots (51). Intriguingly, several fibrinolytic enzymes isolated from earthworms have been identified (52-54), and the regulation of fibrinolytic system components is pathogenetically linked with lipid metabolism (55). The antioxidant properties of EE have also been described by multiple studies $(26,56,57)$. Antioxidants neutralize free radicals produced from liver metabolism and maintain redox homeostasis (58). Patients with NAFLD have increased oxidative stress, largely due to the augmented flux of FFAs to the liver (59). Oxidative stress contributes to the progression of several liver diseases, including AFLD and NAFLD (60). Lirussi et al. attempted to evaluate the beneficial and harmful effects of antioxidant supplementation in patients with NAFLD and found significant attenuation of AST levels compared to levels in the subjects with placebo intervention (61). Therefore, the antioxidant activities of EE may be partly responsible for the reduced liver injury observed in our study.

A glycolipoprotein mixture referred to as G-90 was isolated from earthworms and contains insulin-like growth factor (IGF) as well as epidermal growth factor (EGF) (62). 
IGF is involved not only in glucose metabolism (63) but also in the regulation of lipid metabolism (64). IGF-1 stimulates FFA transport (65) and oxidation (66) in skeletal muscle. Subcutaneous injection of IGF-1 and growth hormone (GH) reduced total plasma cholesterol levels in rats (64); IGF-1 deficiency led to deregulated lipid metabolism and an impaired lipid profile (64). Similarly, EGF has been implicated in the regulation of hepatic and plasma lipid levels in mice (67). Hence, the regulation of the lipid profile and hepatic morphological changes by EE in HFD-fed guinea pigs could be due to the components mentioned above, but further investigation needs to be performed to validate the active ingredients involved and their specific functions.

AST and ALT are transaminases abundantly present in the liver, and they help the liver convert proteins into energy. When the liver sustains injury, AST and ALT are released into the bloodstream, causing an increase in the blood levels of these enzymes (10). It is worth noting that hepatotoxic effects of lipid-lowering drugs such as statins have been reported in patients, with increases in transaminase levels detected $(68,69)$. In our study, significant elevations in AST and ALT were observed in HFD-fed guinea pigs, while EE treatment suppressed the induction of transaminases. These results support the therapeutic potential of EE in treating NAFLD in two aspects: first, EE can reduce the liver damage caused by HFD; second, the dosage of EE used in the current study does not cause significant hepatotoxicity. Hence, EE may be a relatively safe alternative medication compared to prescription drugs. However, it has been reported that elevated serum levels of AST and ALT can also be due to cardiac disease (70) or skeletal muscle damage (71). Therefore, we could not rule out the possibilities of these non-hepatic causes of AST/ ALT changes in our study.

A potential limitation of this study is that we cannot ensure that data obtained from guinea pigs are clinically relevant and that the results mimic the aspects of human disease. Accordingly, we plan to use commercially available human hepatocytes as an in vitro model in the future to investigate the effect of $\mathrm{EE}$ on lipid metabolism and the underlying molecular mechanisms.

\section{Conclusions}

In conclusion, the results of our study strongly indicate that EE can improve the lipid profile and attenuate liver damage in HFD-fed guinea pigs. Although the specific molecules involved await identification, we have demonstrated that $\mathrm{EE}$ has the potential to serve as a relatively safe alternative medication to treat NAFLD one day.

\section{Acknowledgments}

Funding: This work was supported by National Key R\&D Program of China (2019YFA0111900), National Natural Science Foundation of China (81874030, 81902303, 81902233), Provincial Natural Science Foundation of Hunan (2020JJ3060), Guangdong Basic and Applied Basic Research Foundation (2020A151501048), Clinical Research Project of Shenzhen Second People's Hospital (20173357201814, 20203357007, 20203357028), Innovation-Driven Project of Central South University (2020CX045), Undergraduate Innovation Training Program of Central South University (XCX20190545, XCX20190606), the Key program of Health Commission of Hunan Province Wu Jieping Medical Foundation (20201902) and CMA.Young and Middle-aged Doctors Outstanding Development Program--Osteoporosis Specialized Scientific Research Fund Project (G-X-20191107-12).

\section{Footnote}

Reporting Checklist: The authors have completed the ARRIVE reporting checklist. Available at http://dx.doi. org/10.21037/atm-20-5362

Data Sharing Statement: Available at http://dx.doi. org/10.21037/atm-20-5362

Conflicts of Interest: All authors have completed the ICMJE uniform disclosure form (available at http://dx.doi. org/10.21037/atm-20-5362). The authors have no conflicts of interest to declare.

Ethical Statement: The authors are accountable for all aspects of the work in ensuring that questions related to the accuracy or integrity of any part of the work are appropriately investigated and resolved. The study was approved by the Ethics Committee at the First Hospital Affiliated to Shenzhen University (SZU), Shenzhen Second People's Hospital (No. 20170135) granted by institutional/ regional/national ethics/committee/ethics board of the Institutional Animal Care and Use Committee of the SZU, in compliance with national/institutional guidelines for the 
care and use of animals.

Open Access Statement: This is an Open Access article distributed in accordance with the Creative Commons Attribution-NonCommercial-NoDerivs 4.0 International License (CC BY-NC-ND 4.0), which permits the noncommercial replication and distribution of the article with the strict proviso that no changes or edits are made and the original work is properly cited (including links to both the formal publication through the relevant DOI and the license). See: https://creativecommons.org/licenses/by-nc-nd/4.0/.

\section{References}

1. Younossi ZM, Koenig AB, Abdelatif D, et al. Global epidemiology of nonalcoholic fatty liver disease-Metaanalytic assessment of prevalence, incidence, and outcomes. Hepatology 2016;64:73-84.

2. Lam B, Younossi ZM. Treatment options for nonalcoholic fatty liver disease. Therap Adv Gastroenterol 2010;3:121-37.

3. Perdomo CM, Fruhbeck G, Escalada J. Impact of Nutritional Changes on Nonalcoholic Fatty Liver Disease. Nutrients 2019;11:677.

4. Chatrath H, Vuppalanchi R, Chalasani N. Dyslipidemia in patients with nonalcoholic fatty liver disease. Semin Liver Dis 2012;32:22-9.

5. Fabbrini E, Sullivan S, Klein S. Obesity and nonalcoholic fatty liver disease: biochemical, metabolic, and clinical implications. Hepatology 2010;51:679-89.

6. Diehl AM. Fatty liver, hypertension, and the metabolic syndrome. Gut 2004;53:923-4.

7. Fernandez ML, Volek JS. Guinea pigs: a suitable animal model to study lipoprotein metabolism, atherosclerosis and inflammation. Nutr Metab (Lond) 2006;3:17.

8. Aromolaran AS, Colecraft HM, Boutjdir M. High-fat dietdependent modulation of the delayed rectifier $\mathrm{K}(+)$ current in adult guinea pig atrial myocytes. Biochem Biophys Res Commun 2016;474:554-9.

9. Yang R, Guo P, Song X, et al. Hyperlipidemic guinea pig model: mechanisms of triglyceride metabolism disorder and comparison to rat. Biol Pharm Bull 2011;34:1046-51.

10. McGill MR. The past and present of serum aminotransferases and the future of liver injury biomarkers. EXCLI J 2016;15:817-28.

11. Sattar N, Forrest E, Preiss D. Non-alcoholic fatty liver disease. BMJ 2014;349:g4596.

12. Garg A, Simha V. Update on dyslipidemia. J Clin Endocrinol Metab 2007;92:1581-9.
13. Alves-Bezerra M, Cohen DE. Triglyceride Metabolism in the Liver. Compr Physiol 2017;8:1-8.

14. Elshourbagy NA, Meyers HV, Abdel-Meguid SS. Cholesterol: the good, the bad, and the ugly - therapeutic targets for the treatment of dyslipidemia. Med Princ Pract 2014;23:99-111.

15. Fotakis P, Kothari V, Thomas DG, et al. AntiInflammatory Effects of HDL (High-Density Lipoprotein) in Macrophages Predominate Over Proinflammatory Effects in Atherosclerotic Plaques. Arterioscler Thromb Vasc Biol 2019;39:e253-72.

16. Wierzbicki AS, Hardman TC, Viljoen A. New lipidlowering drugs: an update. Int J Clin Pract 2012;66:270-80.

17. Ramkumar S, Raghunath A, Raghunath S. Statin Therapy: Review of Safety and Potential Side Effects. Acta Cardiol Sin 2016;32:631-9.

18. Florentin M, Liberopoulos EN, Elisaf MS. Ezetimibeassociated adverse effects: what the clinician needs to know. Int J Clin Pract 2008;62:88-96.

19. Stoekenbroek RM, Hartgers ML, Rutte R, et al. PCSK9 inhibitors in clinical practice: Delivering on the promise? Atherosclerosis 2018;270:205-10.

20. Jose J. Statins and its hepatic effects: Newer data, implications, and changing recommendations. J Pharm Bioallied Sci 2016;8:23-8.

21. Stolk MF, Becx MC, Kuypers KC, et al. Severe hepatic side effects of ezetimibe. Clin Gastroenterol Hepatol 2006;4:908-11.

22. Castellote J, Ariza J, Rota R, et al. Serious drug-induced liver disease secondary to ezetimibe. World J Gastroenterol 2008;14:5098-9.

23. Tjandrawinata RR, Yunaidi DA, Susanto LW. The Safety and Tolerability of Lumbrokinase DLBS1033 in Healthy Adult Subjects. Drug Res (Stuttg) 2016;66:293-9.

24. Deng Z, Gao S, Xiao X, et al. The effect of earthworm extract on mice S180 tumor growth and apoptosis. Biomed Pharmacother 2019;115:108979.

25. Kawakami T, Fujikawa A, Ishiyama Y, et al. Protective effect of composite earthworm powder against diabetic complications via increased fibrinolytic function and improvement of lipid metabolism in ZDF rats. Bioscience, Biotechnology, and Biochemistry 2016;80:1980-9.

26. Balamurugan $M$, Parthasarathi $K$, Ranganathan LS, et al. Hypothetical mode of action of earthworm extract with hepatoprotective and antioxidant properties. J Zhejiang Univ Sci B 2008;9:141-7.

27. Prakash M, Gunasekaran G, Elumalai K. Effect of earthworm powder on antioxidant enzymes in alcohol 
induced hepatotoxic rats. Eur Rev Med Pharmacol Sci 2008;12:237-43.

28. Parwanto M, Mahyunis, Senjaya H, et al. Fractionation and Characterization of Proteins in Lumbricus rubellus Powders. Inter J Pharm Clini Res 2016;8:15-21.

29. Kleiner DE, Brunt EM, Van Natta M, et al. Design and validation of a histological scoring system for nonalcoholic fatty liver disease. Hepatology 2005;41:1313-21.

30. Brunt EM, Kleiner DE, Wilson LA, et al. Nonalcoholic fatty liver disease (NAFLD) activity score and the histopathologic diagnosis in NAFLD: distinct clinicopathologic meanings. Hepatology 2011;53:810-20.

31. Podell BK, Ackart DF, Richardson MA, et al. A model of type 2 diabetes in the guinea pig using sequential dietinduced glucose intolerance and streptozotocin treatment. Dis Model Mech 2017;10:151-62.

32. Tveden-Nyborg P, Birck MM, Ipsen DH, et al. Dietinduced dyslipidemia leads to nonalcoholic fatty liver disease and oxidative stress in guinea pigs. Transl Res 2016;168:146-60.

33. Riggs SM. Guinea pigs. In: Mitchell MA, Tully TN. Manual of exotic pet practice. Elsevier 2009:456-73.

34. Nutrient Requirements of the Guinea Pig. Nutrient Requirements of Laboratory Animals. Washington (DC): National Academies Press (US), 1995.

35. Ipsen DH, Rolin B, Rakipovski G, et al. Liraglutide Decreases Hepatic Inflammation and Injury in Advanced Lean Non-Alcoholic Steatohepatitis. Basic Clin Pharmacol Toxicol 2018;123:704-13.

36. Rabe H. Reference ranges for biochemical parameters in guinea pigs for the Vettest(R)8008 blood analyzer] Tierarztl Prax Ausg K Kleintiere Heimtiere 2011;39:170-5.

37. Fernandez ML. Guinea pigs as models for cholesterol and lipoprotein metabolism. J Nutr 2001;131:10-20.

38. Toshikuni N, Tsutsumi M, Arisawa T. Clinical differences between alcoholic liver disease and nonalcoholic fatty liver disease. World J Gastroenterol 2014;20:8393-406.

39. Osna NA, Donohue TM, Jr., Kharbanda KK. Alcoholic Liver Disease: Pathogenesis and Current Management. Alcohol Res 2017;38:147-61.

40. Smith BW, Adams LA. Non-alcoholic fatty liver disease. Crit Rev Clin Lab Sci 2011;48:97-113.

41. Benedict M, Zhang X. Non-alcoholic fatty liver disease: An expanded review. World J Hepatol 2017;9:715-32.

42. Kazantzis M, Stahl A. Fatty acid transport proteins, implications in physiology and disease. Biochim Biophys Acta 2012;1821:852-7.

43. Liu Q, Bengmark S, Qu S. The role of hepatic fat accumulation in pathogenesis of non-alcoholic fatty liver disease (NAFLD). Lipids Health Dis 2010;9:42.

44. Sun Z, Miller RA, Patel RT, et al. Hepatic Hdac3 promotes gluconeogenesis by repressing lipid synthesis and sequestration. Nat Med 2012;18:934-42.

45. Sears B, Perry M. The role of fatty acids in insulin resistance. Lipids Health Dis 2015;14:121.

46. Kakimoto PA, Kowaltowski AJ. Effects of high fat diets on rodent liver bioenergetics and oxidative imbalance. Redox Biol 2016;8:216-25.

47. Yu D, Chen G, Pan M, et al. High fat diet-induced oxidative stress blocks hepatocyte nuclear factor 4alpha and leads to hepatic steatosis in mice. J Cell Physiol 2018;233:4770-82.

48. Phan TT, Ta TD, Nguyen DT, et al. Purification and characterization of novel fibrinolytic proteases as potential antithrombotic agents from earthworm Perionyx excavatus. AMB Express 2011;1:26.

49. Ng B, Chanabun R, Panha S. Biological and physiological responses of Perionyx excavatus to abamectin. Environ Sci Pollut Res Int 2019;26:28309-18.

50. Prakash M, Gunasekaran G. Antibacterial activity of the indigenous earthworms Lampito mauritii (Kinberg) and Perionyx excavatus (Perrier). J Altern Complement Med 2011;17:167-70.

51. Wun TC. Plasminogen activation: biochemistry, physiology, and therapeutics. Crit Rev Biotechnol 1988;8:131-48.

52. Nakajima N, Ishihara K, Sugimoto $M$, et al. Chemical modification of earthworm fibrinolytic enzyme with human serum albumin fragment and characterization of the protease as a therapeutic enzyme. Biosci Biotechnol Biochem 1996;60:293-300.

53. Zhao J, Pan R, He J, et al. Eisenia fetida protease-III-1 functions in both fibrinolysis and fibrogenesis. J Biomed Biotechnol 2007;2007:97654.

54. Mihara H, Sumi H, Yoneta T, et al. A novel fibrinolytic enzyme extracted from the earthworm, Lumbricus rubellus. Jpn J Physiol 1991;41:461-72.

55. Brown SL, Sobel BE, Fujii S. Attenuation of the synthesis of plasminogen activator inhibitor type 1 by niacin. A potential link between lipid lowering and fibrinolysis. Circulation 1995;92:767-72.

56. Cao Q, Steinman AD, Yao L, et al. Toxicological and biochemical responses of the earthworm Eisenia fetida to cyanobacteria toxins. Sci Rep 2017;7:15954.

57. Grdisa M, Popovic M, Hrzenjak T. Glycolipoprotein extract (G-90) from earthworm Eisenia foetida exerts 
some antioxidative activity. Comp Biochem Physiol A Mol Integr Physiol 2001;128:821-5.

58. Bocci V, Valacchi G. Free radicals and antioxidants: how to reestablish redox homeostasis in chronic diseases? Curr Med Chem 2013;20:3397-415.

59. Soardo G, Donnini D, Domenis L, et al. Oxidative stress is activated by free fatty acids in cultured human hepatocytes. Metab Syndr Relat Disord 2011;9:397-401.

60. Muriel P, Gordillo KR. Role of Oxidative Stress in Liver Health and Disease. Oxid Med Cell Longev 2016;2016:9037051.

61. Lirussi F, Azzalini L, Orando S, et al. Antioxidant supplements for non-alcoholic fatty liver disease and/or steatohepatitis. Cochrane Database Syst Rev 2007;2007:CD004996.

62. Cooper EL, Hrzenjak TM, Grdisa M. Alternative sources of fibrinolytic, anticoagulative, antimicrobial and anticancer molecules. Int J Immunopathol Pharmacol 2004;17:237-44.

63. Clemmons DR. Metabolic actions of insulin-like growth factor-I in normal physiology and diabetes. Endocrinol Metab Clin North Am 2012;41:425-43, vii-viii.

64. Pascual M, Larralde J, Martinez JA. Insulin-like growth factor I (IGF-I) affects plasma lipid profile and inhibits the lipolytic action of growth hormone $(\mathrm{GH})$ in isolated adipocytes. Life Sci 1995;57:1213-8.

Cite this article as: Deng Z, Gao S, An Y, Huang Y, Liu H, Zhu W, Lu W, He M, Xie W, Yu D, Li Y. Effects of earthworm extract on the lipid profile and fatty liver induced by a highfat diet in guinea pigs. Ann Transl Med 2021;9(4):292. doi: 10.21037/atm-20-5362
65. Heron-Milhavet L, Haluzik M, Yakar S, et al. Musclespecific overexpression of CD36 reverses the insulin resistance and diabetes of MKR mice. Endocrinology 2004;145:4667-76.

66. Leung KC, Ho KK. Stimulation of mitochondrial fatty acid oxidation by growth hormone in human fibroblasts. J Clin Endocrinol Metab 1997;82:4208-13.

67. Scheving LA, Zhang X, Garcia OA, et al. Epidermal growth factor receptor plays a role in the regulation of liver and plasma lipid levels in adult male mice. Am J Physiol Gastrointest Liver Physiol 2014;306:G370-81.

68. Bradford RH, Shear CL, Chremos AN, et al. Expanded Clinical Evaluation of Lovastatin (EXCEL) study results: two-year efficacy and safety follow-up. Am J Cardiol 1994;74:667-73.

69. Wlodarczyk J, Sullivan D, Smith M. Comparison of benefits and risks of rosuvastatin versus atorvastatin from a meta-analysis of head-to-head randomized controlled trials. Am J Cardiol 2008;102:1654-62.

70. Ewid M, Sherif H, Allihimy AS, et al. AST/ALT ratio predicts the functional severity of chronic heart failure with reduced left ventricular ejection fraction. BMC Res Notes 2020;13:178.

71. Nathwani RA, Pais S, Reynolds TB, et al. Serum alanine aminotransferase in skeletal muscle diseases. Hepatology $2005 ; 41: 380-2$. 